\section{SAT0664 CARTILAGE DAMAGE QUANTITATIVELY ASSESSED BY MRI USING T2 MAPPING IN CCP-POSITIVE RA PATIENTS}

N. Renner ${ }^{1}$, A. Kleyer ${ }^{2}$, G. Krönke², D. Simon ${ }^{2}$, J. Rech ${ }^{3}$, G. Schett ${ }^{2}$, G. Welsch ${ }^{4}$ M.L. Pachowsky ${ }^{1}{ }^{1}$ Trauma and Orthopaedic Surgery; ${ }^{2}$ Department of Internal Medicine 3, Rheumatology and Immunology; ${ }^{3}$ Department of Internal Medicine 3, Rheumatolgy and Immunology, Friedrich-Alexander-University Erlangen-Nürnberg (FAU) and Universitätsklinikum Erlangen, Erlangen; ${ }^{4}$ UKE Athleticum, University Hospital Hamburg-Eppendorf, Hamburg, Germany

Background: T2 mapping is a non-invasive quantitative MRI approach which reflects cartilage hydration and collagen integrity without using contrast enhancement. It is a sensitive tool to determine cartilage damage and is currently spreading into broader clinical application. ${ }^{1}$

Objectives: This study aimed to analyse biochemical changes in cartilage of Metacarpophalangeal (MCP) joints in patients with rheumatoid arthritis (RA) using T2 mapping in a 3 T MRI setting.

Methods: Thirty RA patients fulfilling the 2010 ACR/EULAR criteria were recruited. Image acquisition was performed using two surface coils in a 3 T MRI whole body scanner (VERIO; SIEMENS Healthcare). ${ }^{2}$ We obtained high-resolution images of the 2nd and 3rd MCP joint. T2 maps were calculated using a pixelwise, mono-exponential, non-negative least-squares-fit analysis. Region-of-interest (ROI) analysis was performed by dividing the cartilage into medial, central and lateral phalangeal (med, cent, lat $\mathrm{P}$ ) and metacarpal (MC) area. ACPA positive patients were compared to ACPA negative patients. Statistical evaluation was performed by means of univariate ANOVA testing with random factors. A pvalue $<0.05$ was considered statistically significant. The study protocol was approved by the local ethics commission. Written informed consent was obtained from all patients

Results: Fourteen ACPA positive (3 male/11 female) and 16 ACPA negative patients (6 male/10 female) were included. Mean age, sex distribution and disease duration were comparable (age: $49.0 \pm 15.1$ years (ACPA+) vs. $56.1 \pm 10.9$ years (ACPA-), $t=1.494, p=0.146$; sex distribution: $\chi 2(1)=0.918, p=0.338$, disease duration: $7.9 \pm 7.3$ years $(A C P A+)$ vs. $3.9 \pm 3.8$ years (ACPA-), $U=69.0, Z=-1.799$, $\mathrm{p}=0.072$ ).

T2 values were significantly higher in the majority of the ROIs in ACPA-positive (+) RA patients compared to ACPA negative (-) patients with a statistical significance in five of the six ROls.

T2 values in ms: $M C$ med (+) $41.3 \pm 15.3$ vs. (-) $29.6 \pm 7.4 p=0.010$, cent (+) 43.8 \pm 14.6 vs. $(-) 28.4 \pm 10.9, p=\mathbf{0 . 0 0 1}$, lat $(+) 45.6 \pm 13.8$ vs. $(-) 32.0 \pm 8.6, p=0.008$.

P: med (+) $30.3 \pm 14.6$ vs. (-) $25.7 \pm 14.0, p=0.169$, cent (+) $29.9 \pm 13.0$ vs. (-) 22.5 $\pm 10.1, p=0.014$, lat $(+) 37.0 \pm 15.9$ vs. $(-) 28.8 \pm 12.6, p=0.039$.

Conclusions: T2 mapping is a clinically feasible non-invasive MR tool for cartilage evaluation of RA patients' MC joints. ACPA positive RA patients showed significantly increased T2 values compared to ACPA negative patients reflecting a more severe cartilage alteration despite comparable disease duration.

\section{REFERENCES:}

[1] Welsch GH, Apprich S, Zbyn S, Mamisch TC, Mlynarik V, Scheffler K, Bieri $\mathrm{O}$, Trattnig S: Biochemical (T2, T2* and magnetisation transfer ratio) MRI of knee cartilage: feasibility at ultra-high field (7T) compared with high field (3T) strength. European radiology 2011;21(6):1136-1143.

[2] Renner N, Kronke G, Rech J, Uder M, Janka R, Lauer L, Paul D, Herz B, Schlechtweg P, Hennig FF, et al. Anti-citrullinated protein antibody positivity correlates with cartilage damage and proteoglycan levels in patients with rheumatoid arthritis in the hand joints. Arthritis \& rheumatology (Hoboken, NJ) 2014;66(12):3283-3288.

Disclosure of Interest: None declared

DOI: 10.1136/annrheumdis-2018-eular.2849

\section{SAT0665 \\ SYNOVITIS AS A PREDICTOR OF EROSIONS IN THE HAND OSTEOARTHRITIS}

O. Balueva ${ }^{1}$, A. Sarapulova ${ }^{2}$, O. Teplyakova ${ }^{2} .{ }^{1}$ Ultrasound Diagnosis, Medical Association "New hospital"; ${ }^{2}$ Urals state Medical University, Yekaterinburg, Russian Federation

Background: The formation of bone erosions in hand osteoarthritis $(\mathrm{HOA})$ is the result of the association of destructive and inflammatory changes in the joint and leads to a more aggressive course of the disease. X-ray examination assesses destructive bone changes, but there is no possibility to assess the condition of periarticular tissues and inflammatory changes objectively. Visualisation of both erosions and synovitis is possible during ultrasound examination of the hands. On the basis of this results it is possible to predict the further development of the disease.

Objectives: To determine the risk of erosion appearance and the features of progression in patients with hand osteoarthritis in a prospective study.

Methods: The study included 45 women which were $45-75$ years old with the diagnosis of HOA, defined by criteria of the American College of Rheumatologists (ACR). 22 joints were examined at each patient by ultasound: distal interphalangeal joints (DIP), proximal interphalangeal joints (PIP), joints of the thumb and $1 \mathrm{st}$ carpo-metacarpal joint (1 st CMC). Ultrasound examination included multiplane gray-scale investigation of the listed above groups of joints. The presence and severity of synovial hypertrophy and effusion followed by evaluation of the degree of synovitis was determined in the B-mode; the quantity and the sizes of osteophytes and bone erosions were evaluated in a similar way. The vascularisation of synovial hypertrophy was estimated in the power Doppler ultrasound. The study was started in 2013. In 3 years the study was repeated. The results were processed with STATISTICA 10.0 software (StatSoft Inc. USA) using the methods of nonparametric statistics. For the description of communication of qualitative signs we used coefficient "odds ratio" OR and $\chi 2$. The results were recognised statistically reliable at value $p<0.05$.

Results: The total number of hand joints was evaluated using ultrasound 990 . In the primary examination 391 joints had synovitis, 46 joints had synovitis and erosion. In 3 years the number of joints with synovitis and the number of joints with synovitis and erosion increased up to 443 and 73 respectively. In 2016 among 73 joints with synovitis and erosion, 40 joints were with erosion against the background of synovitis 3 years ego, and only in the 8 joints erosion were revealed where was no synovitis. Thus the frequency of erosion detection in hand joints at repeated examination was higher in the cases the synovitis was detected at primary examination: $\chi 2=16.38 \mathrm{p}=0.0001$; OR 4.373, Cl 95\% 2.01-9.47.

Conclusions: The synovitis in hand joints is a prognostic adverse factor of erosions appearance and transformation in the erosive subtype of osteoarthritis. The possibility one-time visualisation of inflammatory and destructive changes allows using ultrasound investigation at primary and differential diagnostics, and dynamic evaluation.

Disclosure of Interest: None declared

DOI: 10.1136/annrheumdis-2018-eular.1702

\section{SAT0666 INFLAMATION BEYOND CLINICAL REMISSION: ULTRASOUND AS A TOOL TO GUIDE US TO REMISSION}

P. Estrada ${ }^{1}$, C. Diaz-Torné2 ${ }^{2}$, V. Torrente ${ }^{3}$, P. Moya ${ }^{2}$, C. Moragues ${ }^{4}$, D. De La Fuente $^{5}$, S. Ros ${ }^{5}$, J. Ramirez ${ }^{6}$, M. Moreno ${ }^{7}$, E. García ${ }^{8}$, M. Puyol ${ }^{9}$, N. Busquets ${ }^{10}$, A. Ponce ${ }^{10}$, J. De Agustín ${ }^{11}$ on behalf of Ecocat on behalf of ECOCAT. ${ }^{1} \mathrm{Hospital}$ Moisès Broggi-CSl; ${ }^{2} \mathrm{Hospital}$ de la Santa Creu i Sant Pau; ${ }^{3} \mathrm{Hospital}$ General de L'Hospitalet, ${ }^{4}$ Hospital Plató; ${ }^{5}$ Hospital Viladecans; ${ }^{6}$ Hospital Clínic; ${ }^{7}$ Hospital Parc Taulí; ${ }^{8}$ Hospital Mollet, ${ }^{9}$ Mútua de Terrassa; ${ }^{10}$ Hospital Granollers; ${ }^{11}$ Hospital de la Vall d'Hebron, Barcelona, Spain

Background: Among patients with RA in remission, subclinical synovitis (SS) has a prevalence of $45 \%$ and is associated with an increased risk of clinical relapse and progression to structural damage. US is a sensitive and accessible tool for evaluating $\mathrm{SS}^{1,2,3}$

Objectives: To analyse US as a tool for evaluating SS in RA patients treated with tocilizumab (TCZ), in order to assess remission, and from there on propose therapeutic tapering.

Methods: Multicenter, 1 year follow up study in 45 patients with RA treated with TCZ. The project was aproved by Ethics Committees and all the patients gave their informed consent. At each visit: DAS28, SDAl, CDAI, mHAQ, US grey scale (GS) and Power Doppler (PD) parameters for 32 joints $(\mathrm{J})$ and 28 tendons $(T)$, with a semiquantitative scale from $0-3$ points. A quantitative index was obtained for $J$ and $T$ in $G S$ and $P D$ and overall (EG +PD) for each patient/visit. SS was considered as the presence of synovitis with $\mathrm{PD}(+) \geq 2$. Our intra and interobserver kappa index was 0.8 .

Results: A significant reduction of all clinical indexes and US variables was observed in all patients. Patients were divided into two groups: remission $(R)$ and no remission (NR) according to whether they achieved DAS28 $\leq 2.6$ at 12 months. Group R achieved DAS28 $\leq 2.6$ after mo 3, whereas US showed SS (GS+, PD >2) until mo 12. The final overall PD value in the R group was $0.6( \pm 0.9)$. 

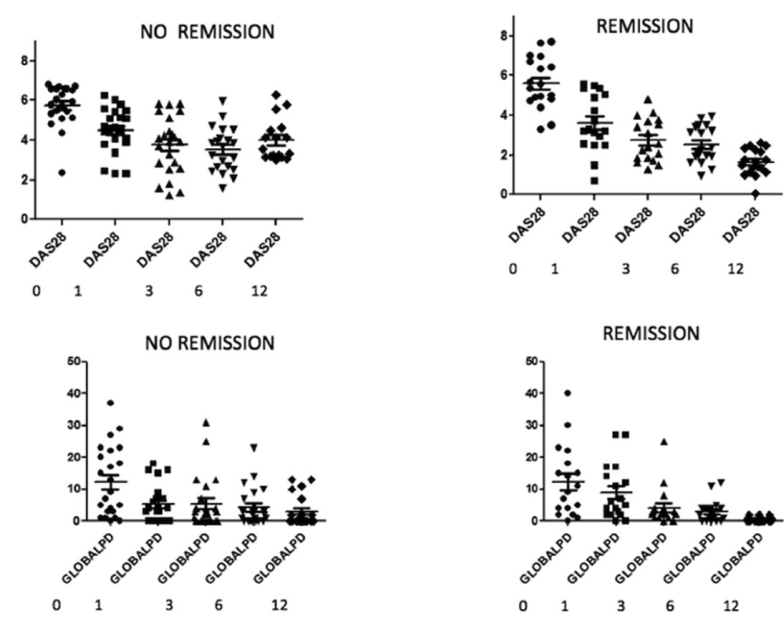

Conclusions: A significant number of patients achieve clinical remission within a few months of starting TCZ. The R group, achieved a good EULAR response since mo 3; a progressive improvement in PD, persisted until mo 12. We consider that these findings refer to SS. From our data we consider that dose tapering should not be initiated until at least 9 months from the start of remission.

\section{REFERENCES:}

[1] doi 10.1002/art.22190

[2] PMID 27050636

[3] doi 10.1093/rheumatology/kex084

Disclosure of Interest: None declared DOI: 10.1136/annrheumdis-2018-eular.4295

\section{SAT0667 IMPLEMENTATION AND ROLE OF MODERN MUSCULOSKELETAL IMAGING IN RHEUMATOLOGICAL PRACTICE IN EUROPE}

P. Mandl ${ }^{1}$, A. Ciechomska ${ }^{2}$, X. Baraliakos ${ }^{3}$, P.G. Conaghan ${ }^{4}$, M.-A. D'Agostino ${ }^{5}$, A. lagnocco ${ }^{6}$, C.J. van der Laken ${ }^{7}$, M. Ostergaard ${ }^{8}$, L. Terslev ${ }^{8}$, E. Naredo ${ }^{9}$.

${ }^{1}$ Rheumatology, Medical University Vienna, Vienna, Austria; ${ }^{2}$ Rheumatology, University Hospital Wishaw, Wishaw, UK; ${ }^{3}$ Rheumazentrum Ruhrgebiet, RuhrUniversity Bochum, Herne, Germany; ${ }^{4}$ Leeds Institute of Rheumatic and Musculoskeletal Medicine, University of Leeds, Leeds, UK; ${ }^{5}$ Rheumatology, APHP, Hôpital Ambroise Paré, Boulogne-Billancourt, France; ${ }^{6}$ Dipartimento di Scienze Cliniche e Biologiche, Università degli Studi di Torino, Turin, Italy, ${ }^{7}$ Rheumatology, VU University Medical Center, Amsterdam, Netherlands; ${ }^{8}$ Center for Rheumatology and Spine Diseases, Copenhagen University Hospital Glostrup, Copenhagen, Denmark; ${ }^{9}$ Rheumatology, Joint and Bone Research Unit, Hospital Universitario Fundación Jiménez Díaz and Autónoma University, Madrid, Spain

Background: Modern non-X-ray imaging methods are increasingly utilised by rheumatologists though the uptake and use of these techniques has developed very differently across Europe and there is limited data on their present impact in rheumatology for individual countries.

Objectives: To document the current implementation, role and training in modern musculoskeletal imaging techniques: musculoskeletal ultrasound (MSUS), magnetic resonance imaging (MRI), computed tomography (CT), and positron emission tomography (PET) among rheumatologists in the member countries of the European League Against Rheumatism (EULAR).

Methods: A EULAR Taskforce comprised of 9 rheumatologist experts in imaging developed English-language questionnaires for each imaging modality (CT, MRI, MSUS, PET) which were sent out to: national rheumatology societies of EULAR, national societies of the European Federation of Societies for Ultrasound in Medicine and Biology, the European Society for Magnetic Resonance Ultrasound in Medicine and Biology, as well as identified experts in the given modalities involved in research and/or training. The surveys were distributed via an online survey tool (Surveymonkey). Simple descriptive and summary statistics were calculated from the responses.

Results: A total of 205 out of 395 experts replied to the surveys. More than $90 \%$ of MSUS experts had an MSUS unit in their department. The majority of responders reported having easy access to MRI, CT or PET (56\%, $78 \%$ and $50 \%$ respectively). Suspicion of rheumatoid arthritis and peripheral spondyloarthrity were the main clinical indications for performing MSUS for diagnostic purposes. Suspicion of sacroilitis and degenerative spine disease were the most common indications to perform MRI or CT for diagnostic purposes, while PET was mainly performed to diagnose large vessel vasculitis and investigation of fever of unknown origin. When asked about the percentage of rheumatologists performing MSUS in their country $37 \%$ of experts reported less than $20 \%, 33 \%$ reported values between $20 \%-40 \%$ and $24 \%$ reported more than $40 \%$ (6\%: unknown). The overwhelming majority (99\%) of experts were certified in MSUS, while only $22 \%$ and $26 \%$ of experts in MRI and CT were certified in their techniques. Seventy-seven percent of responders reported that their national rheumatology societies organise MSUS courses, while courses in MRI or CT organised by the national rheumatology societies were less commonly reported (38\% and $16 \%$ respectively).

Conclusions: Rheumatologists in Europe utilise modern imaging techniques, however access among the techniques and training offered is varied.

Disclosure of Interest: None declared

DOI: 10.1136/annrheumdis-2018-eular.4911

\section{SAT0668 ARTICULAR INVOLVEMENT IN CHRONIC HEPATITIS C INFECTION - A PRELIMINARY ULTRASOUND STUDY}

R.A. Ionescu, ${ }^{1,2}$, L.M. Pana ${ }^{3}$, V.M. Pompilian ${ }^{1}$, A. Voicu ${ }^{4}$, S. Caraiola', D. Ramba ${ }^{3}$, C.R. Baicus ${ }^{5}$, A. Dan ${ }^{5} .{ }^{1} 3$ rd Internal Medicine Dept, "Carol Davila" University of Medicine and Pharmacy; ${ }^{2}$ Colentina Clinical Hospital, Bucharest, Romania; ${ }^{3} 3 r d$ Internal Medicine Dept, Colentina Clinical Hospital; " "Alexandru Obregia"Clinical Hospital; ${ }^{5}$ Internal Medicine Clinic, "Carol Davila" University of Medicine and Pharmacy, Bucharest, Romania

Background: Chronic hepatitis $C$ is a public health issue. Extra-hepatic manifestations of this infection also include articular involvement, that sometimes mimics rheumatoid arthritis.

Objectives: Evaluation of potential articular involvement in patients diagnosed with chronic hepatitis $\mathrm{C}$ infection and possible correlations with biological abnormalities.

Methods: We evaluated 31 patients with chronic hepatitis $\mathrm{C}$ infection, using ultrasound (US) examination of both wrists and the second and fifth interphalangeal joints of the fingers. Then, we looked for possible correlations with various laboratory findings.

Results: $29 \%$ of patients were symptomatic (pain) at the time of the US examination.

$10 \%$ of our patients had tenosynovitis of the extensor tendons, another $10 \%$ had active synovial hypertrophy (Doppler positive), $31.14 \%$ had inactive synovia hypertrophy, $20.9 \%$ had erosions and in $20 \%$ of them the US examination of the above mentioned joints was normal.

$61.29 \%$ of patients having US abnormalities, where rheumatoid factor positive. All patients with erosions and inactive synovial hypertrophy, $83.3 \%$ of those with active synovial hypertrophy and $67 \%$ of those with tenosynovitis where cryoglobulins positive.

We found low levels of $\mathrm{C} 3$ fraction of complement in 92.3 patients with erosions, 83.3 patients with active synovial hypertrophy, 84.2 patients wit inactive synovial hypertrophy and $50 \%$ of patients with tenosynovitis.

In $85.7 \%$ of patients with active synovial hypertrophy, and $53.8 \%$ of pastients with erosions, the C-reactive protein (CRP) levels where elevated.

No patient was diagnosed as also having rheumatoid arthritis

Conclusions: Even in asymptomatic patients with chronic hepatitis $\mathrm{C}$ infection US examination of the wrists and of the second and fifth interphalangeal joins may reveal abnormalities. Certain types of these are probably correlated with the presence of cryoglobulins and possibly associated with CRP levels, C3 levels and rheumatoid factors positivity.

Further studies are needed to verify our finding

Disclosure of Interest: None declared

DOI: 10.1136/annrheumdis-2018-eular.7464

\section{SAT0669 99MTC-HDP SPECT/CT - A NEW METHOD IN EARLY DIAGNOSIS OF SACROILEITIS}

R.A. Hemelein ${ }^{1}$, Z. Besenyi ${ }^{2}$, A. Bakos ${ }^{2}$, L. Kovács ${ }^{1}$, L. Pávics ${ }^{2} .{ }^{1}$ Department of Rheumatology and Immunology, ${ }^{2}$ Department of Nuclear Medicine, University of Szeged, Szeged, Hungary

Background: 99Tc hydroxyl-disphophonate SPECT-CT (HDP SPECT-CT) is a hybrid imaging technology, which is a new modality in the examination of the sacroiliac joint. It is a high resolution, low dose structural computer tomography scan fused with a functional metabolism recording. Our aim was to investigate whether it is comparable in sensitivity with MRI, to analyse quantitatively the radioisotope uptake, to assess its potential in the assessment of inflammatory activity, and in the diagnosis of spondylarthitis (SpA).

Objectives: Seventeen patients ( 9 women, 8 men, mean age 35 years) were involved into the study from July 2016 . The patients were selected according the following clinical features: inflammatory type low back pain raising the suspicion of axial SpA,${ }^{17}$ elevated CRP level, ${ }^{6}$ HLA-B27 positivity, ${ }^{8}$ associated oligo- or 